\title{
A simple method of drying polyacrylamide slab gels that eliminates cracking
}

Ghanshyam D Heda*, 1 (iD

${ }^{1}$ Department of Sciences \& Mathematics, Mississippi University for Women, Columbus, MS 39701, USA; *Author for correspondence: gdheda@muw.edu

BioTechniques 70: 55-57 (January 2021) 10.2144/btn-2020-0117

First draft submitted: 28 July 2020; Accepted for publication: 2 October 2020; Published online: 23 November 2020

\section{ABSTRACT}

The polyacrylamide slab gel is the most common gel format for analyzing protein samples by electrophoresis. Drying these gels is useful in many biological applications; for example, autoradiography, in which radiolabeled proteins are separated to enable their detection and identification. Dried protein gels can also serve as an ideal method of preserving the gel itself for permanent record-keeping and allowing densitometry at a convenient time. Here I describe a simple and highly reproducible gel-drying method that results in dried gels without the cracks that are frequently encountered with many existing gel-drying methods.

\section{METHOD SUMMARY}

After staining and destaining, polyacrylamide slab gels are presoaked in a gel-drying buffer, sandwiched between cellophane sheets and assembled with an acrylic plate with several holes and a frame. A hypodermic needle is inserted into air pockets around the gel edges to vent trapped air and allow the gel to air-dry on the laboratory bench.

\section{KEYWORDS:}

gel cracking • gel drying • polyacrylamide gel $\bullet$ SDS-PAGE • sieve acrylic plate

Since its introduction by Ornstein [1] and Davis [2], polyacrylamide gel electrophoresis has become the most common analytical method used in the study of proteins. The slab format is most popular, because it enables uniformity in simultaneously electrophoresing multiple samples and is convenient for both one- and two-dimensional PAGE. Slab gels can be dried for record-keeping or used in autoradiography, fluorography, transmission densitometry, display on an overhead projector and future applications like extraction of proteins for sequencing. Several methods have been described for drying slab gels. Almost all these methods can be categorized into one of two groups:

- Air-drying methods, in which gels are sandwiched between cellophane or plastic sheets and passively dried at ambient temperature [37], with the help of fans [8], or in an oven at $45-70^{\circ} \mathrm{C}$ [9]. The gel sandwich is prepared with cellophane or plastic sheets that are supported either by plates and frames [3-9] or by having weights placed on them [10-12].

- Heat-drying methods, in which gels are dried using a commercially available heater and vacuum pump [13-15].

Although air-drying methods are slow, they are widely used because they are simple and cost-effective. While heat-drying methods are rapid, they require expensive equipment and routine maintenance. A common problem with both methods is the cracking of gels, which is primarily caused by rapid dehydration, by air trapped around the edges of the gel between the cellophane sheets, or (in the case of heat-drying methods) by overheating or disruption of electricity.

Here I describe an improvement to a standard gel-drying method in which the gel is sandwiched between two cellophane sheets and supported using either two acrylic frames [3,4] or an acrylic frame and a plate [5-9]. Cellophane sheets are cellulose-based and porous, enabling evaporation from the gel. The method using two frames exposes the gel to atmospheric air on both sides and allows more rapid drying. The method using a frame on one side and a solid plate on the other exposes the gel to atmospheric air on only one side and thereby extends drying time. Regardless of the length of drying time, a major drawback with these methods is that gels can easily crack due to rapid dehydration of the gel (especially when it is exposed to air on both sides) and/or air trapped around the edges of the gel between the cellophane sheets, which can slowly migrate into the gel body as the gel dehydrates. To overcome these problems, some simple modifications have been made to the procedure.

Instead of using two frames or a frame and a solid plate, this modified method uses one frame and a plate with several holes (referred to herein as a sieve acrylic plate, SAP). The square plate and a square frame with a $\sim 1.5-\mathrm{cm}$ border can be cut out of an acrylic material such as Plexiglass ${ }^{\circledR}$. Several holes of roughly $1-2 \mathrm{~mm}$ in diameter can be drilled into the square plate to create the SAP. As shown in Figure 1B, specially designed clamps or binder clips can be used in assembling the gel-cellophane sandwich. The SAP and a frame of 


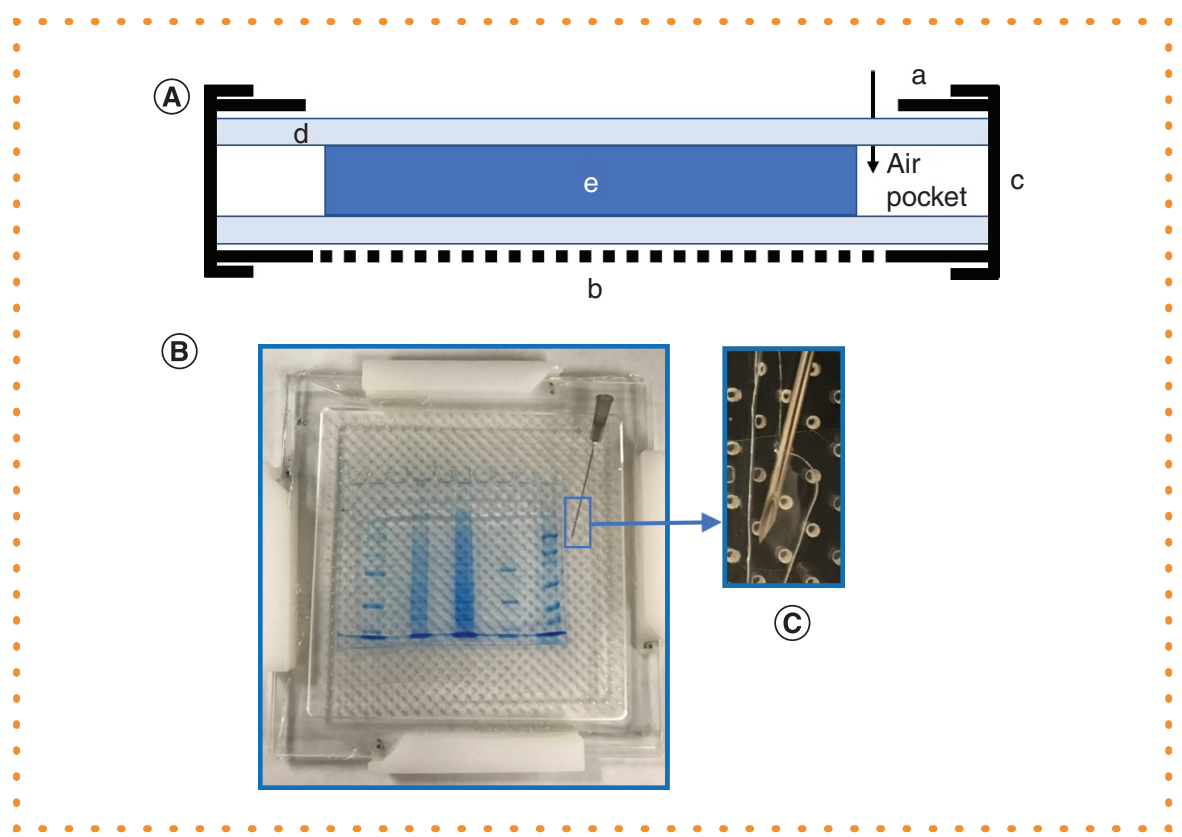

Figure 1. Gel-drying assembly. (A) Schematic diagram of the gel-drying assembly. (a) Acrylic frame; (b) sieve acrylic plate; (c) clamps; (d) cellophane sheets; (e) polyacrylamide gel. A hypodermic needle is inserted at the arrow for venting air that is trapped around the edges of the gel. (B) Top view of an actual assembly containing a gel to be dried. White clamps shown in the figure can be replaced by regular binder clips. (C) Magnified view of a portion of gel-drying assembly (B) showing a hypodermic needle inserted into air pockets at the edge of gel in between the two cellophane sheets.

size $15 \times 15 \mathrm{~cm}$ is suitable for drying a mini-gel of dimensions $8 \times 8 \mathrm{~cm}$. For larger gels, larger plates can be used.

Mammalian cell lysates and protein MW markers were electrophoresed on gels containing $7.5 \%$ polyacrylamide or a $4-15 \%$ linear gradient of polyacrylamide by SDS-PAGE using a standard procedure [16]. The gels were stained with AcquaStain, a single-step Coomassie G-250 blue protein stain (Bulldog Bio, NH, USA) and then destained with water. Destained gels ready to be dried were soaked for $\sim 1 \mathrm{~h}$ in a gel-drying solution consisting of $30 \%$ methanol and $5 \%$ glycerol, which can be prepared in the lab or purchased commercially from vendors such as Bio-Rad (CA, USA) or Thermo Fisher Scientific (MA, USA). A gel assembly is shown in Figure 1, ready for drying according to the following protocol:

- Trim two sheets of cellophane (Diversified Biotech, MA, USA) to about 1-2 cm wider on each side than the SAP. Wet these sheets with the gel-drying solution in a plastic or glass container.

- Place one of the sheets on the SAP (Figure 2A), then put the presoaked gel onto this sheet, followed by another sheet of cellophane on top of the gel.

- Remove any trapped air from this gel-cellophane sandwich (Figure 1C) by finger pressure and/or rolling a pipette over it.

- Lay the acrylic frame (Figure 2B) on top of the gel-cellophane sandwich.

- Holding the assembly in one hand, stretch both layers of cellophane sheets with the other hand, pull them over the SAP and start putting clamps on the entire assembly one side at a time.

- Carefully insert a single 22- or 18-gauge needle anywhere into the air pocket around the edges of the gel.

- Leave the entire assembly on a laboratory bench at ambient room temperature for air-drying (Figure 1B).

- With the help of a magnifying glass, examine the gel body for tiny trapped air bubbles. If any are found, invert the assembly (turn it SAP side up) and add drops of gel-drying solution in the area where air bubbles were noticed; leave the assembly in this position. Gel-drying solution added to the holes of the SAP slowly enters the gel through the porous cellophane sheet, replaces the air bubbles and prevents cracking of the gel.

Polyacrylamide gels sandwiched between cellophane sheets and assembled with the SAP and a frame as described above prevented gel cracking by slowing the speed of gel dehydration and extending the gel drying time compared with methods using two frames. Other authors have dehydrated gels by presoaking in $65 \%$ methanol and $0.5 \%$ glycerol [9], but this method generally results in gel shrinkage. Regardless of the number of frames and the type of plates used, these procedures did not eliminate the problem of air trapped around the edges of the gel and the cellophane sheets. As the gel dehydrates, this trapped air can move toward the center of the gel slab and cause cracks. To release this trapped air, a 22- or 18-gauge hypodermic needle was carefully inserted in the air pocket surrounding the gel edges, allowing air to vent through the needle as the gel air-dries by dehydration (Figure 1B \& C). The combination of these two 


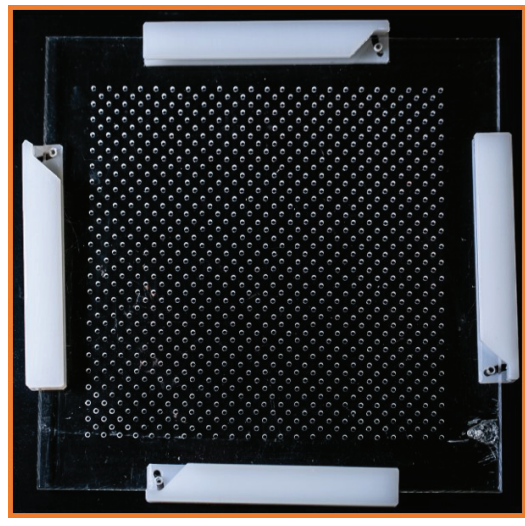

(A)

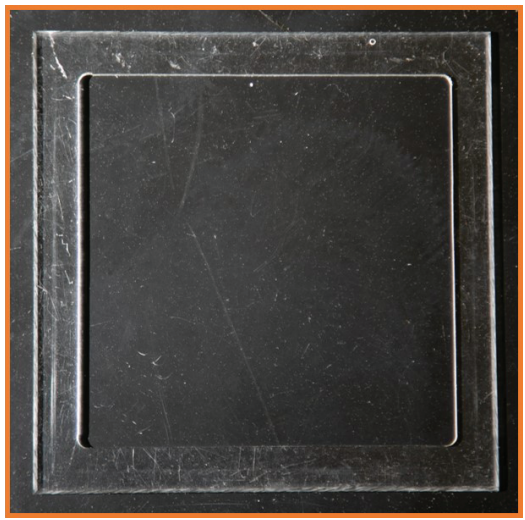

(B)

Figure 2. Gel-drying device. (A) $15 \times 15 \mathrm{~cm}$ sieve acrylic plate with several holes of approximately $1 \mathrm{~mm}$ diameter each. Specially designed white clamps shown in the figure can be replaced by regular binder clips. (B) $15 \times 15 \mathrm{~cm}$ acrylic frame with a border of approximately $1.25 \mathrm{~cm}$ width.

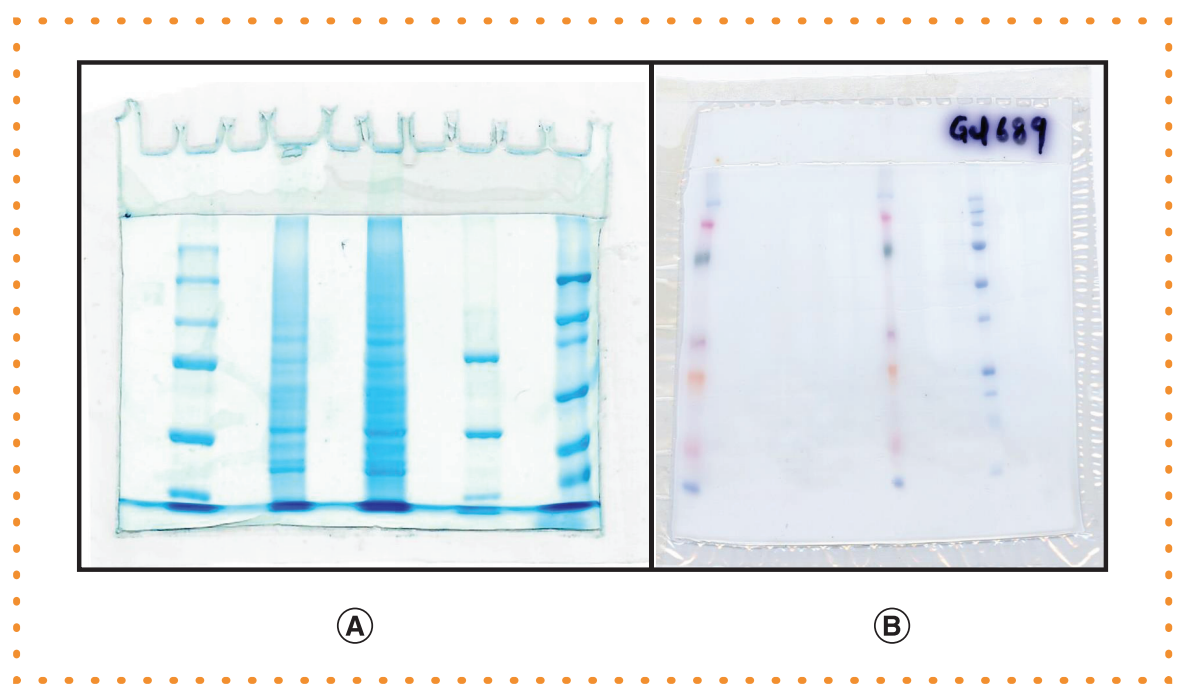

Figure 3. Dried polyacrylamide gels. (A) 7.5\% SDS-PAGE; and (B) 4-15\% gradient SDS-PAGE. These gels were air-dried using the gel-drying assembly shown in Figure 1, and as per the method described.

modifications (i.e., using SAP and venting trapped air using a hypodermic needle) resulted in dried gels that not only were free of cracks but also were softer and maintained their original size (Figure 3). Some of the low-abundance protein bands appeared sharper and more 'scannable' in dried gels compared with wet gels. Dried gels are finally removed from the gel-drying unit, trimmed, wrapped with soft tissue and kept under a heavy book $(\sim 5-10 \mathrm{~kg})$ for about $2 \mathrm{~h}$ to prevent curling. The dried gel then can be attached to the laboratory notebook for record-keeping.

Besides storage, gels dried by this method are useful in autoradiography and densitometry; however, they may not be useful in fluorography because both sides of the gel are permanently attached to the cellophane sheets. Instead, a layer of plastic food wrap can be inserted on one side between the cellophane and the gel so that it can be peeled to expose the gel to $\mathrm{x}$-ray film, as described [7,8]. Further, gradient gels are generally difficult to dry compared with gels of uniform acrylamide concentration. However, we found that this modified method is useful in drying gels of both types (Figure 3).

The gel-drying method described here normally takes about 24-36 h for complete drying, depending on the concentration and thickness of the polyacrylamide gels. For example, a $1.5-\mathrm{mm}$ thick $7.5 \%$ polyacrylamide gel can dry in about $24 \mathrm{~h}$, whereas gels of the same thickness with a 4-20\% polyacrylamide gradient may take up to $36 \mathrm{~h}$ to dry completely. Gels with a thickness of only $1 \mathrm{~mm}$ dry faster. This method therefore takes slightly longer than the method in which two frames are used $[3,4]$ but is faster than the method using one 
frame and a solid plate [5-7]. This modified gel-drying method is cost-effective, produces mostly crack-free dried gels and is a good compromise in terms of gel drying time.

We routinely use this modified method of gel drying for obtaining soft and crack-free gels with uniform concentrations or linear gradients of polyacrylamide. Future studies will determine whether drying of gels by this method can be expedited using electric fans [8] or hot-air ovens [9].

\section{Author contributions}

$\mathrm{G}$ Heda conceived the plan and designed and conducted the experiments.

\section{Acknowledgments}

The author would like to thank his wife R Heda for useful suggestions, and C Jenkins (Mississippi University for Women) for some of the photographs.

\section{Financial \& competing interests disclosure}

This work was supported by the Mississippi INBRE and funded by an Institutional Development Award (IDeA) from the National Institute of General Medical Sciences of the NIH (grant no. P20GM103476) and by faculty research awards from Mississippi University for Women and Mississippi-NASA to G Heda. The author has no other relevant affiliations or financial involvement with any organization or entity with a financial interest in or financial conflict with the subject matter or materials discussed in the manuscript apart from those disclosed.

No writing assistance was utilized in the production of this manuscript.

\section{Open access}

This work is licensed under the Attribution-NonCommercial-NoDerivatives 4.0 Unported License. To view a copy of this license, visit http: //creativecommons.org/licenses/by-nc-nd/4.0/

\section{References}

1 Ornstein L. Disc electrophoresis I. Background and theory. Ann. NY Acad. Sci. 121, 321-349 (1964).

2 Davis BJ. Disc electrophoresis II. Method and application to human serum proteins. Ann. NY Acad. Sci. 121, 404-426 (1964).

3 Wallevik K, Jensenius JC. A simple and reliable method for the drying of polyacrylamide slab gels. J. Biochem. Biophys. Methods 6(1), 17-21 (1982).

4 Feldman A, Berndt N, Bergmann R, Bachmann M. Gel drying methods. Methods Mol. Biol. 1853, 269-271 (2018).

5 Michaels JD, Ford JC. Inexpensive method for air-drying polyacrylamide electrophoresis gels. BioTechniques 11(4), 466-467 (1991).

6 Wiedbrauk DL, Zielinski SA, Nehls S. An inexpensive gel-drying system. BioTechniques 11(4), 470-472 (1991).

7 Porter DR, Gatschet MJ. Simplified drying of polyacrylamide gels for fluorography. BioTechniques 13(3), 363-364 (1992).

8 Krishnan M, Nguyen HT. Drying acrylamide slab gels for fluorography without using gel drier and vacuum pump. Anal. Biochem. 87(1), 51-53 (1990).

9 Juang RH, Chang YD, Sung HY, Su JC. Oven-drying method for polyacrylamide gel slab packed in cellophane sandwich. Anal. Biochem. 141(2), 348-350 (1984).

10 Samal BB. Drying and storage of polyacrylamide slab gels: a simple procedure. Anal. Biochem. 163(1), 42-44 (1987).

11 Turner RJ, Taylor DE, Rothery RA, Weiner JH. Easy method for SDS-PAGE gel drying and preservation. BioTechniques 14(2), 200-201 (1993).

12 Guervaziev YV. Polyacrylamide gel fixation by drying and gluing to both paper and transparent plastic. BioTechniques 27(2), 294 (1999).

13 Skinner MK, Griswold MD. Fluorographic detection of radioactivity in polyacrylamide gels with 2,5-diphenyloxazole in acetic acid and its comparison with existing procedures. Biochem. J. 209(1), 281-284 (1983)

14 Cheng ML, VandeBerg JL. A versatile technique for drying polyacrylamide slab gels. J. Biochem. Biophys. Methods 11(2-3), 137-143 (1985).

15 Smith BJ. Drying gels. Methods Mol. Biol. 32, 157-161 (1994).

16 Laemmli UK. Cleavage of structural proteins during the assembly of the head of bacteriophage T4. Nature 227(5259), 680-685 (1970). 